Theoretical and experimental study on electron interactions with chlorobenzene:

Shape resonances and differential cross sections

Alessandra Souza Barbosa, Márcio T. do N. Varella, Sergio d'A. Sanchez, João Ameixa, Francisco Blanco, Gustavo García, Paulo Limão-Vieira, Filipe Ferreira da Silva, and Márcio H. F. Bettega'

Citation: The Journal of Chemical Physics 145, 084311 (2016); doi: 10.1063/1.4961649

View online: http://dx.doi.org/10.1063/1.4961649

View Table of Contents: http://aip.scitation.org/toc/jcp/145/8

Published by the American Institute of Physics

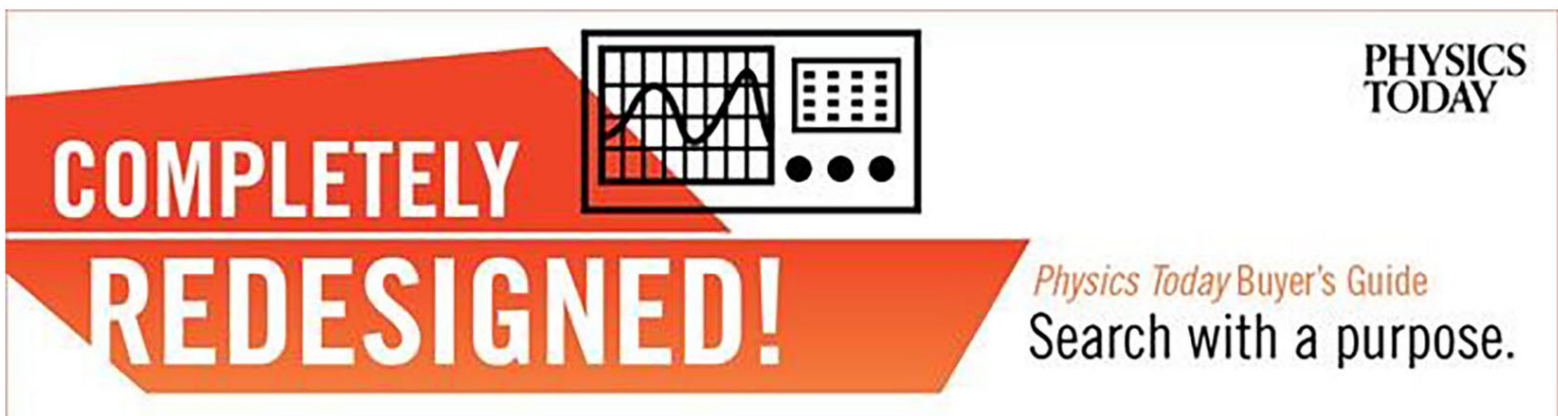




\title{
Theoretical and experimental study on electron interactions with chlorobenzene: Shape resonances and differential cross sections
}

\author{
Alessandra Souza Barbosa, ${ }^{1,2}$ Márcio T. do N. Varella, ${ }^{3}$ Sergio d'A. Sanchez, ${ }^{1}$ \\ João Ameixa, ${ }^{2}$ Francisco Blanco, ${ }^{4}$ Gustavo García, ${ }^{5}$ Paulo Limão-Vieira, ${ }^{2}$ \\ Filipe Ferreira da Silva, ${ }^{2}$ and Márcio H. F. Bettega ${ }^{1, a)}$ \\ ${ }^{1}$ Departamento de Física, Universidade Federal do Paraná, CP 19044, 81531-990 Curitiba, Paraná, Brazil \\ ${ }^{2}$ Laboratório de Colisões Atómicas e Moleculares, CEFITEC, Departamento de Física, Faculdade de Ciências \\ e Tecnologia, Universidade Nova de Lisboa, 2829-516 Caparica, Portugal \\ ${ }^{3}$ Instituto de Física, Universidade de São Paulo, Rua do Matão 1731, 05508-090 São Paulo, SP, Brazil \\ ${ }^{4}$ Departamento de Física Atómica, Molecular y Nuclear, Facultad de Ciencias Físicas, \\ Universidad Complutense de Madrid, E-28040 Madrid, Spain \\ ${ }^{5}$ Instituto de Física Fundamental, Consejo Superior de Investigaciones Científicas (CSIC), Serrano 113-bis, \\ 28006 Madrid, Spain
}

(Received 30 May 2016; accepted 29 July 2016; published online 31 August 2016)

\begin{abstract}
In this work, we report theoretical and experimental cross sections for elastic scattering of electrons by chlorobenzene ( $\mathrm{ClB})$. The theoretical integral and differential cross sections (DCSs) were obtained with the Schwinger multichannel method implemented with pseudopotentials (SMCPP) and the independent atom method with screening corrected additivity rule (IAM-SCAR). The calculations with the SMCPP method were done in the static-exchange (SE) approximation, for energies above $12 \mathrm{eV}$, and in the static-exchange plus polarization approximation, for energies up to $12 \mathrm{eV}$. The calculations with the IAM-SCAR method covered energies up to $500 \mathrm{eV}$. The experimental differential cross sections were obtained in the high resolution electron energy loss spectrometer VG-SEELS 400, in Lisbon, for electron energies from $8.0 \mathrm{eV}$ to $50 \mathrm{eV}$ and angular range from $7^{\circ}$ to $110^{\circ}$. From the present theoretical integral cross section (ICS) we discuss the low-energy shape-resonances present in chlorobenzene and compare our computed resonance spectra with available electron transmission spectroscopy data present in the literature. Since there is no other work in the literature reporting differential cross sections for this molecule, we compare our theoretical and experimental DCSs with experimental data available for the parent molecule benzene. Published by AIP Publishing. [http://dx.doi.org/10.1063/1.4961649]
\end{abstract}

\section{INTRODUCTION}

Electron collisions play an important role in many areas, such as low-temperature plasmas, astronomical environments, and in biological systems. In the latter case, the pioneer study of Boudaiffa et al. ${ }^{1}$ indicated that single and double strand breaks can be induced in DNA by low energy electrons (LEEs). It was shown that the underlying dissociation processes can be mediated by electron attachment to vacant valence orbitals, i.e., by the formation of shape resonances, in specific sites of the DNA chains. ${ }^{2}$ Since then, intense theoretical and experimental activity has focused on better understanding the interactions of LEEs with biomolecules and their precursors, ${ }^{3-5}$ in particular the characterization of transient anion states (resonances) and their role in the dissociation processes. More recently, halogenated DNA and RNA bases, ${ }^{6-9}$ as well their precursors, ${ }^{10}$ have also gained attention. In this case, the motivation is the potential radiosensitizing activity of the halogenated species that can be incorporated into the gene sequence and give rise, through the dissociative electron attachment (DEA) mechanism described above, to radicals that subsequently damage the DNA. Once chemically

\footnotetext{
a)Electronic mail: bettega@ fisica.ufpr.br
}

introduced in the organism, the radiosensitizing drugs are preferentially absorbed by the rapidly replicating cancer cells, thus making the radiation therapy more efficient. ${ }^{11}$

Chlorobenzene $(\mathrm{ClB})$ is a prototype for radiosensitizers and also a seemingly simple system with a peculiar spectrum of transient anion states. As in the closely related halopyrimidine molecules, ${ }^{10}$ the halogen substituent gives rise to $\sigma^{*}$ resonances and tends to stabilize the $\pi^{*}$ anion states. ${ }^{10,12}$ Similar trends are expected in CIB, but interesting features also arise from the halogen-induced symmetry breaking, i.e., the lifting of the degeneracy of the $\pi^{*}\left(E_{2 u}\right)$ anion state of benzene located at $1.14 \mathrm{eV}^{13}$ In fact, vibronic effects underlying the dynamics of the $\pi^{*}\left(B_{1}\right)$ and $\pi^{*}\left(A_{2}\right)$ resonances in ClB have been inferred from the available electron transmission spectroscopy (ETS), ${ }^{14,15}$ DEA, ${ }^{14,16-19}$ and electron energy loss spectroscopy (EELS) ${ }^{20}$ data. From their ETS data, Olthoff et al. ${ }^{14}$ assigned shape resonances at $0.73,2.50$, and $4.50 \mathrm{eV}$, in good agreement with the ETS measurements of Burrow et al., ${ }^{15}$ namely, $0.75,2.42$, and $4.39 \mathrm{eV}$. However, the latter authors also discussed a weaker band around $1.15 \mathrm{eV}$. The higher lying signals were assigned to $\sigma_{\mathrm{CCl}}^{*}(2.42 \mathrm{eV})$ and $\pi^{*}(4.39 \mathrm{eV})$ anion states, while the lowest energy signal $(0.75 \mathrm{eV})$ to the nearly degenerate $\pi^{*}\left(B_{1}\right)$ and $\pi^{*}\left(A_{2}\right)$ resonances, such that vibronic effects would lead to the weak 
band at $1.15 \mathrm{eV}$. More recently, Skalický et al..$^{20}$ explored the vibrational dynamics arising from the formation of the $\pi^{*}\left(B_{1}\right)$, $\pi^{*}\left(A_{2}\right)$, and $\sigma_{\mathrm{CCl}}^{*}$ resonances, and discussed both $\pi^{*}\left(B_{1}\right) / \pi^{*}\left(A_{2}\right)$ and $\pi^{*}\left(B_{1}\right) / \sigma_{\mathrm{CCl}}^{*}$ vibronic couplings, among other features. The latter coupling is consistent with the DEA ${ }^{14,16-19}$ data, showing a very intense peak for the elimination of the $\mathrm{Cl}^{-}$anion around $0.7 \mathrm{eV}$. Modelli and Venuti ${ }^{21}$ employed different theoretical methods to compute the energies for the lowest $\pi^{*}$ and $\sigma^{*}$ anion states of ClB and related molecules. In coupled-cluster calculations, they obtained the values of $2.02 \mathrm{eV}, 2.10 \mathrm{eV}$, and $4.07 \mathrm{eV}$ for the $\pi^{*}\left(A_{2}\right), \pi^{*}\left(B_{1}\right)$, and $\sigma_{\mathrm{CCl}}^{*}$ anion states, respectively. Lunt et al. ${ }^{22}$ reported total cross sections (TCSs) at very low collision energies $(<1 \mathrm{eV})$ for halobenzenes, mainly driven by the dipole-allowed rotational excitations. Finally, Makochekanwa et al. ${ }^{23}$ also obtained TCSs for electron and positron collisions with $\mathrm{ClB}$, for impact energies from 0.2 up to $1000 \mathrm{eV}$. These authors assigned the structures located at $0.8 \mathrm{eV}$ and $2.5 \mathrm{eV}$, as expected, but their interpretation of the data was not consistent with the previously reported experimental data outlined above.

In the present work, we report theoretical and experimental cross sections for elastic scattering of electrons by ClB. The theoretical integral (ICS) and differential cross sections (DCS) were calculated with two different methodologies: the Schwinger multichannel method implemented with pseudopotentials (SMCPP) and the independent atom model with screening corrected additivity rule (IAM-SCAR). In the SMCPP calculations, we employed two approximations, namely, the static-exchange (SE) approximation and the staticexchange plus polarization (SEP) approximation. From our calculated ICSs, we could identify the shape resonances of $\mathrm{ClB}$ and compare the results with the available ETS data. ${ }^{14,15}$ We also report experimental DCSs for impact energies ranging from $8 \mathrm{eV}$ to $50 \mathrm{eV}$ and angular range from $7.0^{\circ}$ to $110^{\circ}$ and compare the measurements with the present DCSs computed with the SMCPP and IAM-SCAR methods. Since, to our knowledge, DCSs have not been reported for electron-ClB collisions, it is desirable to compare the present data with DCSs for the parent molecule benzene. ${ }^{24}$

The remainder of this paper is as follows. In Section II, we highlight some relevant points of the two theoretical methods employed in the calculations and in Section III, we present some details of the experimental apparatus. In Section IV, we present and discuss our theoretical and experimental results. And in Section V, we close the paper with a brief summary of the present results.

\section{THEORY}

\section{A. The Schwinger multichannel method implemented with pseudopotentials}

Our calculations were carried out with the Schwinger multichannel method (SMC) $)^{26,27}$ with pseudopotentials. ${ }^{28}$ The method was recently reviewed ${ }^{29}$ and here we will provide only the relevant points for the present calculations.

The SMC method is a variational approximation to the scattering amplitude, so that the resulting expression in the body-frame is given by
$f_{\mathrm{SMC}}\left(\vec{k}_{f}, \vec{k}_{i}\right)=-\frac{1}{2 \pi} \sum_{m, n}\left\langle S_{\vec{k}_{f}}|V| \chi_{m}\right\rangle\left(d^{-1}\right)_{m n}\left\langle\chi_{n}|V| S_{\vec{k}_{i}}\right\rangle$,

where the $\left\{\left|\chi_{m}\right\rangle\right\}$ represents a basis set of $(N+1)$-electron symmetry-adapted Slater determinants, also referred to as configuration state functions (CSFs). The CSFs are built from products of target states with one-particle wave functions. For the calculations carried out in the static-exchange (SE) approximation, the $(N+1)$-electron basis set (direct space) is given by

$$
\left|\chi_{m}\right\rangle=\mathcal{A}\left(\left|\Phi_{1}\right\rangle \otimes\left|\varphi_{m}\right\rangle\right),
$$

where $\left|\Phi_{1}\right\rangle$ is the target ground state, $\left|\varphi_{m}\right\rangle$ is a singleparticle function, and $\mathcal{A}$ is the antisymmetrizer. For the calculations carried out in the static-exchange plus polarization approximation, the direct space is augmented by CSFs constructed as

$$
\left|\chi_{m}\right\rangle=\mathcal{A}\left(\left|\Phi_{r}\right\rangle \otimes\left|\varphi_{s}\right\rangle\right),
$$

where $\left|\Phi_{r}\right\rangle$ are $N$-electron Slater states obtained by performing single excitations of the target from the occupied (hole) orbitals to a set of unoccupied (particle) orbitals. Here $\left|\varphi_{s}\right\rangle$ is also a single-particle function and $\mathcal{A}$ is the antisymmetrizer.

In Eq. (1), the $d_{m n}$ matrix elements are given by

$$
d_{m n}=\left\langle\chi_{m}\left|A^{(+)}\right| \chi_{n}\right\rangle
$$

and the $A^{(+)}$operator is given by

$$
A^{(+)}=\frac{1}{2}(P V+V P)-V G_{P}^{(+)} V+\frac{\hat{H}}{N+1}-\frac{1}{2}(\hat{H} P+P \hat{H}) .
$$

In the above equations, $S_{\vec{k}_{i(f)}}$ is a product of a target state and a plane wave with momentum $\vec{k}_{i(f)}$, which is an eigenstate of the unperturbed Hamiltonian $H_{0} ; V$ is the interaction potential between the incident electron and the target; $\hat{H} \equiv E-H$ is the collision energy minus the full Hamiltonian of the system, with $H=H_{0}+V ; P$ is a projection operator onto the open-channel space; and $G_{P}^{(+)}$is the free-particle Green's function projected on the $P$-space.

The ground state geometry of $\mathrm{ClB},{ }^{31}$ which has $\mathrm{C}_{2 v}$ symmetry, was optimized using the package GAMESS ${ }^{32}$ at the second order Møller-Plesset Perturbation Theory (MP2) and using 6-31 $(d)$ basis set (in Figure 3 it is possible to see the geometrical structure of $\mathrm{ClB}$ ). The ground state of chlorobenzene was described at the Hartree-Fock (HF) level, where the core electrons of $\mathrm{C}$ and $\mathrm{Cl}$ atoms were described employing the norm-conserving pseudopotentials of Bachelet, Hamann, and Schlüter ${ }^{30}$ and the valence target electrons were represented by Cartesian Gaussian functions which were generated according to Ref. 33 and were given elsewhere. ${ }^{10,25}$ The $d$-type orbitals were considered as having five components to avoid linear dependency in the basis set.

To represent particle and scattering orbitals in SEP calculations, we employed the Improved Virtual Orbitals (IVOs). ${ }^{34}$ To generate the IVOs, we used the highest occupied orbital as a hole orbital and a triplet coupling.

We selected IVOs that satisfy the relation ${ }^{35}$

$$
\varepsilon_{\text {par }}-\varepsilon_{\text {hole }}+\varepsilon_{\text {scat }}<\Delta,
$$


where $\varepsilon_{\text {par }}$ is the particle orbital energy, $\varepsilon_{\text {hole }}$ is the hole orbital energy (which was determined employing the Hartree-Fock approximation), $\varepsilon_{\text {scat }}$ is the scattering orbital energy, and $\Delta$ is the energy cut. We used $\Delta=1.76$ hartree and considered all singlet and triplet coupled excitation for all symmetries such that we obtained $11809 \mathrm{CSF}$ for $A_{1}$ symmetry, $11646 \mathrm{CSF}$ for the $B_{2}$ symmetry, $10685 \mathrm{CSF}$ for the $B_{1}$ symmetry, and 10488 CSFs for $A_{2}$ symmetry.

The calculated dipole moment of $\mathrm{ClB}$ is $2.02 \mathrm{D}$, which is about $20 \%$ bigger than the experimental value of $1.69 \mathrm{D} .{ }^{46}$ The SMC method employs only square integrable functions in the expansion of the scattering wave function. When dealing with molecules that possess a permanent electric dipole moment, the long-range character of the dipole potential is truncated by the range of the Cartesian Gaussian functions, and as a consequence, the higher partial waves are not correctly described. To investigate the impact of long-range interactions on the present calculations, we included the dipole potential through a closure procedure described by Oliveira et al. ${ }^{47}$ With this procedure, the lower partial waves of the scattering amplitude are computed with the SMC method (we will call $l_{S M C}$ the highest partial wave considered from the SMC method) and the higher partial waves are computed with a scattering for the dipole moment potential of the molecule computed in the first Born approximation. ${ }^{47}$ The number of lower partial waves included in the calculation depends on the impact energy and is chosen in order to provide the DCSs obtained with and without the Born closure correction in agreement at medium and higher scattering angles. For the calculations in the SEP approximations, we employed $l_{S M C}=3$ for energies up to $1.5 \mathrm{eV}, l_{S M C}=4$ from $1.6 \mathrm{eV}$ to $3.5 \mathrm{eV}, l_{S M C}=5$ from 4.0 to $5.8 \mathrm{eV}, l_{S M C}=6$ from 5.9 to $7.5 \mathrm{eV}$, and $l_{S M C}=7$ from 8.0 to $12 \mathrm{eV}$. At higher energies, in the SE approximation, we employed $l_{S M C}=8,9,10$ at $20 \mathrm{eV}$, $30 \mathrm{eV}$, and $50 \mathrm{eV}$.

\section{B. Independent atom model calculations}

Details of the application of the IAM-SCAR ${ }^{36,37}$ method to electron interactions have been provided in a number of previous papers. ${ }^{38-42}$ Briefly, each atomic target $(\mathrm{C}, \mathrm{Cl}$, $\mathrm{H})$ is represented by an interacting complex potential, the so-called optical potential. The real part accounts for the elastic scattering of the incident electrons, and the imaginary part represents the inelastic processes, which is considered as "absorption" from the incident beam. For the elastic part, the potential is represented by the sum of three terms that include the follwing: (a) a static term derived from a HartreeFock calculation of the atomic charge density distribution, (b) an exchange term to account for the indistinguishability of the incident and target electrons, and (c) a polarization term for the long-range interactions which depends on the target polarizability. The inelastic scattering, on the other hand, is treated as electron-electron collisions. Further improvements to the original formulation in the description of the electron's indistinguishability and the inclusion of screening effects led to a model which provides a good approximation for electron-atom scattering over a broad energy range. To calculate the cross sections for electron collisions with $\mathrm{C}_{3} \mathrm{H}_{6} \mathrm{O}$, the additivity rule (AR) is then applied to the optical model results for each constituent atom. In this approach, the molecular scattering amplitude stems from the coherent sum of all the relevant atomic amplitudes, which gives the DCSs for the molecule of interest. The geometry of the molecule (atomic positions and bond lengths) is taken into account by using some screening coefficients and this enables the range of validity of the technique to be extended down to impact energies of $20 \mathrm{eV}$ for electron scattering.

\section{EXPERIMENTAL APPARATUS AND OPERATING PROCEDURES}

The High Resolution Electron Energy Loss Spectrometer (HREELS) used in the Lisbon laboratory, VG-SEELS 400, has been described in detail elsewhere, ${ }^{43}$ so only a brief discussion will be presented here. A monochromatic electron beam is generated with a hemispherical electron monochromator crossing orthogonally with an effusive molecular beam that enters the interaction region through a hypodermic needle with a $0.95 \mathrm{~mm}$ inner diameter. After the electron interaction with the target gas, the scattered electrons are energy analyzed with a hemispherical electron analyzer, which can rotate about the gas jet, and detected by an electron multiplier. The typical base pressure in the main chamber was $5.0 \times 10^{-5} \mathrm{~Pa}$ and, upon gas admission, this increased to a pressure of $1.0 \times 10^{-3} \mathrm{~Pa}$. The liquid sample was supplied from Sigma-Aldrich with a quoted purity of $99.9 \%$. The sample was degassed by repeated freeze-pump-thaw cycles prior to use.

In the current experiments, the energy resolution of the incident electron beam was $150 \mathrm{meV}$ [full width at half maximum (FWHM)], with incident electron currents of a few nA (depending on the initial electron energy). Such a value means that, in principle, there could be contributions to the elastic signal from some of the lower-lying vibrational modes of $\mathrm{ClB}$. However, in the energy range above $10 \mathrm{eV}$, these possible vibrational contributions are expected to be very small compared to the elastic intensity and thus are not expected to make any significant contribution to the measured elastic cross sections, whereas below $10 \mathrm{eV}$, those have been extracted by deconvoluting the energy loss spectra with Gaussian profiles and separated from the elastic peak. The elastic contribution was carefully fitted with a Gaussian function so that any residual contributions from underlying vibrational modes were separated from the elastic peak. However, contributions from those vibrational modes with energies as low as $150 \mathrm{meV}$ cannot be discarded from the elastic peak. Notwithstanding, and in order to minimize those contributions, careful comparison procedure has been implemented to the experimental elastic DCS trending as a function of the scattering angle with the data provided by the theoretical methods. Such comparison resulted in total agreement between experiment and theory. The calibration of the energy loss scale has been obtained according to the position of the elastic peak. The hemispherical electron analyzer is placed on a turntable stage and can be rotated from $0^{\circ}$ to $+120^{\circ}$, with respect to the incident electron beam, with an angular acceptance of $\left(1.25^{\circ} \pm 0.25^{\circ}\right)$. The incident energy 
of the electron beam was obtained following a methodology described by Khakoo et al. ${ }^{44}$ Briefly, the analyzer was tuned to the elastic peak and the EEL spectrum was carefully monitored to the maximum cutoff in the energy loss spectrum. The incident energy value was then set by proper adjustment of the electron gun acceleration voltage to obtain the required cutoff voltage.

The absolute scale of the present differential cross sections (DCSs) was set by the theoretical results. We further discuss the normalization procedure in Sec. IV. We estimate that the experimental uncertainties on the resulting experimental DCS lie in the range of $15 \%-30 \%$, with the actual value depending on the specific incident electron energy $\left(E_{0}\right)$ and scattered electron angle $(\theta)$ under consideration. This overall error is largely comprised of an uncertainty in keeping the correct flow conditions and, to a much lesser extent, an error associated with the statistical accuracy of the data and the stability of the incident electron beam $(<1 \%)$.

\section{RESULTS}

Figure 1 shows our theoretical ICS obtained with the SMCPP approach in the SEP and SEP+Born approximations, as well as our ICS calculated with the IAM-SCAR method. The present results are compared with the experimental TCS from Refs. 22 and 23. The cross sections present a sharp increase as the incident energy goes to zero, as expected in electron collisions against polar targets. Note that the energy resolution used in Ref. 23 is quite poor, around $0.25 \mathrm{eV}$ in the energy range shown in this figure, and therefore they are not able to resolve dipole interactions within the transmitted electron beam. These processes lead to very low energy, a few meVs on average, rotational excitations which are strongly peaked in the forward direction and consequently they are not accounted for in their experimental conditions. Besides, the wide entrance and exit apertures in the apparatus do not allow to discriminate the scattered from unscattered electrons in the forward direction. These circumstances tend to underestimate the measured total cross sections and especially for the lower energies where the dipole interactions are dominant. As the energy increase, i.e., above $20 \mathrm{eV}$, a better agreement is found between the calculated IAM-SCAR data and the experimental TCS, as expected. The present ICS (SEP+Born) at very low energies is found in reasonable agreement with the high resolution measurements of Lunt et al., ${ }^{22}$ with discrepancies around $30 \%$ below $0.6 \mathrm{eV}$. However, the present SEP+Born calculations differ from the Born-dipole model of Ref. 22, since (i) we account for short-range (other than dipole) interactions in the lower partial waves, which would be important for scattering at higher angles; (ii) our Born corrections employ an overestimated dipole moment magnitude (consistent with the HF description of the target molecule); (iii) our model amounts to an approximate rotationally summed cross section from the rotational ground state of the target, without accounting for thermal averages over the initial rotational states (see Oliveira et $a{ }^{47}$ for details); and (iv) we do not correct the cross sections to account for undetected electrons at low scattering angles as described by Lunt $e t a l .,{ }^{22}$ although we integrate the
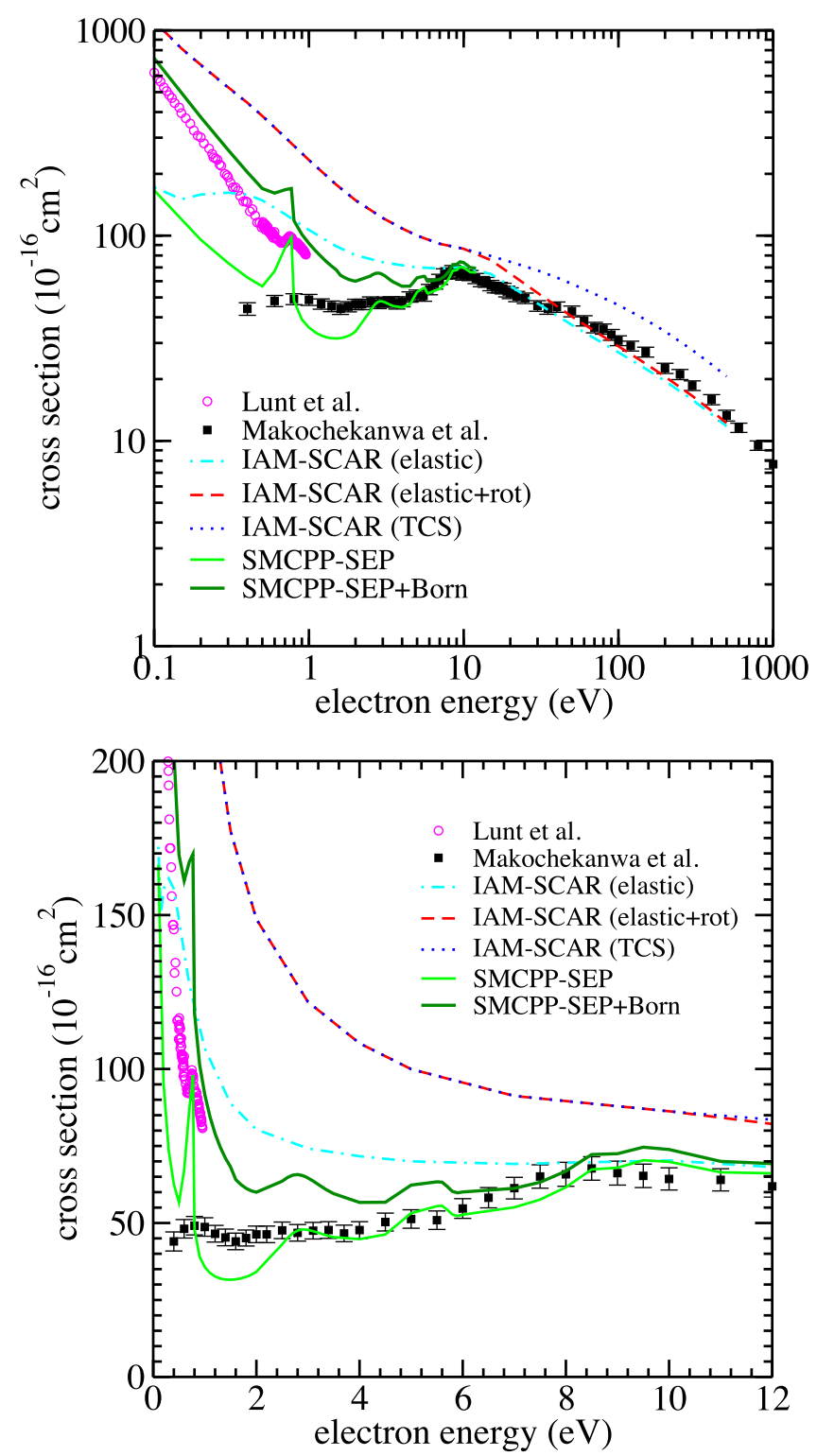

FIG. 1. Integral cross section for electron interactions with chlorobenzene for energies up to $1000 \mathrm{eV}$ (upper panel) and up to $12 \mathrm{eV}$ (lower panel).

DCSs over $1^{\circ} \leq \theta \leq 180^{\circ}$. In any case, the magnitude of the calculated SEP+Born ICS would be more accurate than the TCS reported in Ref. 23, as suggested by the comparison with the data of Lunt et al.

Regarding the resonances, the lowest-lying structure in the SMCPP ICS, about $0.7 \mathrm{eV}$, is related to the nearly degenerate $\pi^{*}\left(B_{1}\right)$ and $\pi^{*}\left(A_{2}\right)$ resonances (see below). The peak position agrees with the experimental $\operatorname{TCS}^{22,23}(0.75$ and $0.8 \mathrm{eV}$, respectively), as well as with the ETS data ${ }^{14,15}$ $(0.73 \mathrm{eV}-0.75 \mathrm{eV})$ and the DEA data ${ }^{14,16-18}(\sim 0.7 \mathrm{eV})$. There is also agreement for the second structure, arising from the $\sigma_{\mathrm{CCl}}^{*}$ anion state, since the peak at $2.8 \mathrm{eV}$ in the SMCPP ICS is consistent with the experimental data $(\sim 2.5 \mathrm{eV})$. The DEA signal for the $\mathrm{Cl}^{-}$fragment is only observed around $0.7 \mathrm{eV}$ (not around $2.5 \mathrm{eV}$ ), indicating that the dissociation arises from an indirect mechanism.

We present in Figure 2 the symmetry decomposition of the SMCPP ICS according to the $C_{2 v}$ group. As expected, the 

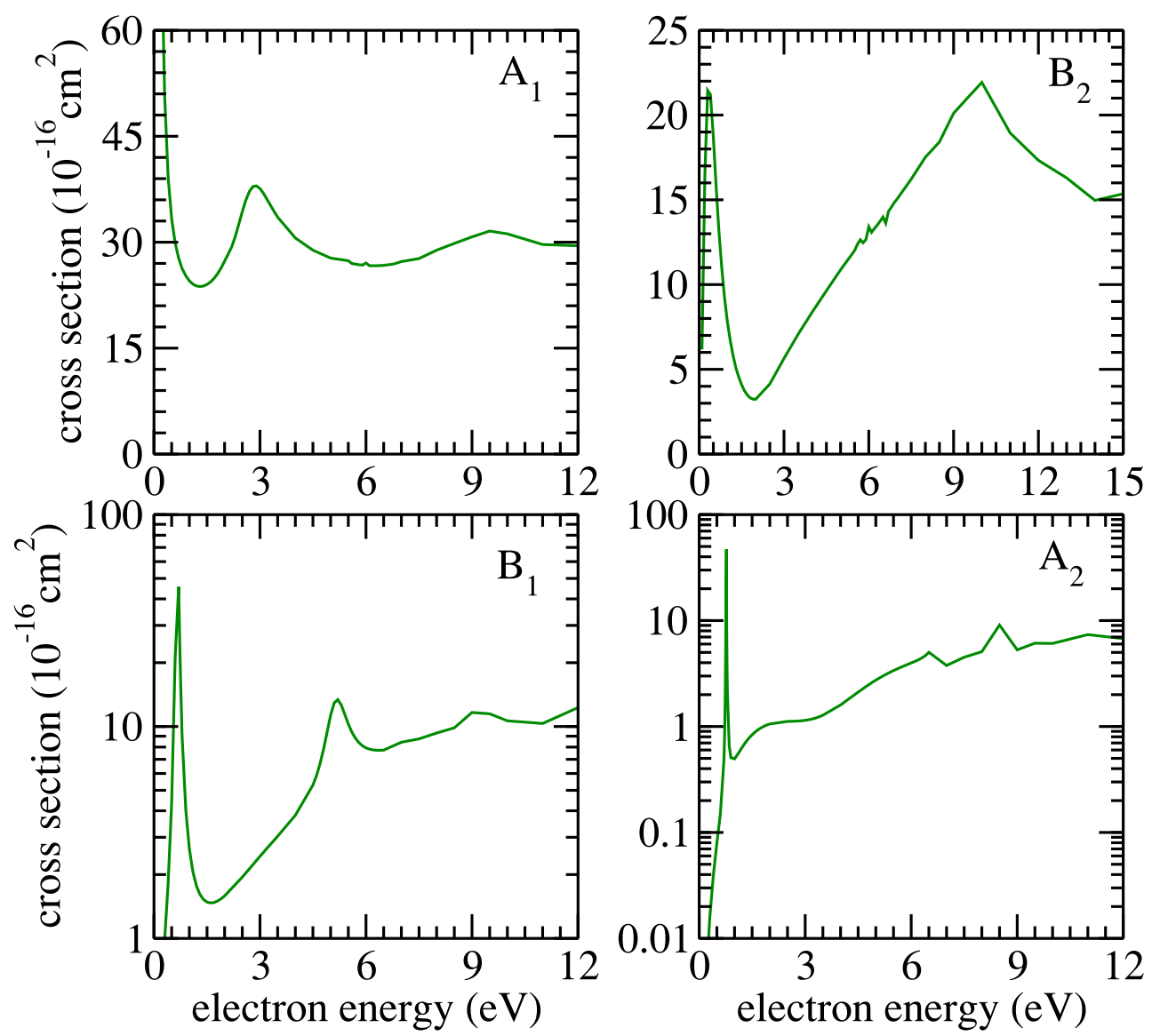

FIG. 2. Symmetry decomposition of the integral cross section for elastic scattering of electrons by chlorobenzene, obtained in the static-exchange plus polarization approximation, according with $C_{2 v}$ point group.

first structure in the ICS arises from overlapping resonances in the $B_{1}(0.70 \mathrm{eV})$ and $A_{2}(0.77 \mathrm{eV})$ symmetries, formed by the occupation of the $\pi^{*}$ LUMO $\left(b_{1}\right)$ and LUMO+1 $\left(a_{2}\right)$ orbitals of $\mathrm{ClB}$, shown in Figure 3. As already observed, the present results are consistent with the experimental data around $0.70 \mathrm{eV}$. However, our fixed-nuclei calculations cannot account for the vibrational structure present in both the electron transmission ${ }^{15}$ and energy loss spectra. ${ }^{20}$ In fact the lack of vibronic couplings in the ICS computations prevents the description of the feature around $1.15 \mathrm{eV}$ in the ETS data. The resonance around $2.8 \mathrm{eV}$ in the $A_{1}$ symmetry arises from electron attachment into the $\sigma_{\mathrm{CCl}}^{*}$ LUMO+2 orbital (see Figure 3 ) and is found in good agreement with the experimental data, as discussed above.

There is also a second structure in the $B_{1}$ symmetry at around $5.20 \mathrm{eV}$, which is also assigned as a $\pi^{*}$ resonance and is related to the LUMO+8 orbital. Although the agreement of this resonance position with the ETS data location of $4.5 \mathrm{eV}$ and with the electron impact spectroscopy value of $4.6 \mathrm{eV}^{20}$ is somewhat poorer than the others resonances, one should note that the description of this resonance is more difficult since it has a mixed character of shape and core-excited resonances. ${ }^{45}$ There is also a broad structure around $10.0 \mathrm{eV}$ in our ICS which agrees well with the TCS data and the ETS measurements that indicate the presence of a resonance at this energy. The empty orbitals, obtained with the $6-31 \mathrm{G}(\mathrm{d})$ basis set, relevant to the formation of the shape resonances discussed here are shown in Figure 3.

When we compare the ETS data of benzene and ClB, we can see that the presence of the chlorine atom brings the position of the $\pi^{*}$ resonances to lower energies, from $1.14 \mathrm{eV}$ and $4.85 \mathrm{eV}$ in benzene ${ }^{13}$ to $0.73 \mathrm{eV}$ and $4.50 \mathrm{eV}$, according to Olthoff et al. ${ }^{14}$ and to $0.75 \mathrm{eV}$ and $4.39 \mathrm{eV}$, according to Burrow et al. ${ }^{15}$ in $\mathrm{ClB}$. Also, a $\sigma^{*}$ resonance is present in the $\mathrm{C}-\mathrm{Cl}$ bond. This is the same effect as observed when comparing halopyrimidines and pyrimidines molecules. ${ }^{10}$ There are at least two previous works available in the literature reporting cross sections for low-energy electron scattering by benzene. ${ }^{50,51}$ Especially, we highlight the $a b$ initio calculation performed by Bettega et al. ${ }^{50}$ also using the SMC method, which placed the $\pi^{*}$ resonances at $2.23 \mathrm{eV}$ and $8.39 \mathrm{eV}$, with the first resonance being double degenerate. In this work, we see that the breakage of symmetry due to the chlorine atom "splits" the first resonance of benzene into two, as expected, even though the experimental data are unable to observe this. Also, our resonance positions are in a much lower energy position when compared to the benzene calculation. One aspect that can account for this large difference, even though both calculations used the SMC method, is the different approaches used in the polarization of the target and a much bigger number of configurations used in the present work. However, the difference in energy of the $\pi^{*}$ resonances is also seen in the experimental 

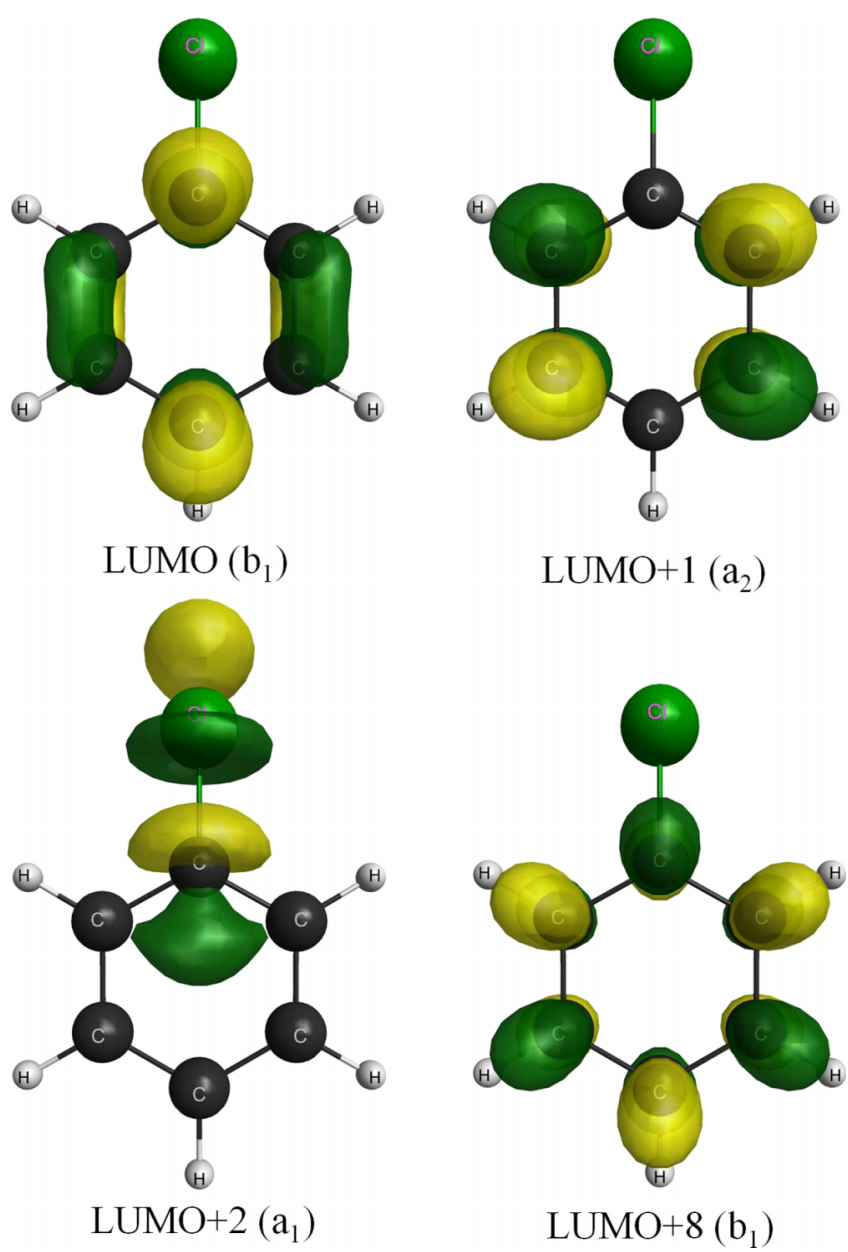

FIG. 3. Empty orbitals, obtained from a HF/6-31G(d) calculation, relevant for the description of the low-energy resonances of chlorobenzene.

data. Hence, in order to observe why the presence of the chlorine atom would force the $\pi^{*}$ shape resonances to lower energies, even though our Figure 3 shows no participation of this atom in the resonance state, we performed net charge calculation using two different basis sets, namely, 6-31G $(d)$ and $\mathrm{TZV}++(2 d, 1 p)$, and the package GAMESS, ${ }^{32}$ in which we compared the net charge of the ring and the isolated chlorine atom. What we observed is that, due to its electronegativity, the chlorine atom makes the ring more positive than for the isolated benzene ring. Hence, it is expected that the resonance positions are shifted to lower energies due to a more attractive potential, as previously pointed out. ${ }^{22,23}$ However, Makochekanwa et al. ${ }^{23}$ inferred that the resonances around $0.8 \mathrm{eV}$ and $2.5 \mathrm{eV}$ in their TCS would arise from the splitting of the $E_{2 u}$ anion states of benzene, due to the halogen substitution. We argue that the first structure located at $0.8 \mathrm{eV}$ corresponds to the nearly degenerate $\pi^{*}$ resonances (in the $B_{1}$ and $A_{2}$ symmetries), while the structure at $2.5 \mathrm{eV}$ corresponds to the $\sigma^{*}$ resonance, in consistency with Refs. 14 , 15 , and 22 .

In Figure 4, we present the calculated differential cross sections, obtained with the SMCPP method in the SEP approximation at 3.0, 4.0, and $6.0 \mathrm{eV}$ compared with experimental data available for benzene molecule. ${ }^{24}$ The poor agreement between both sets of data is explained,
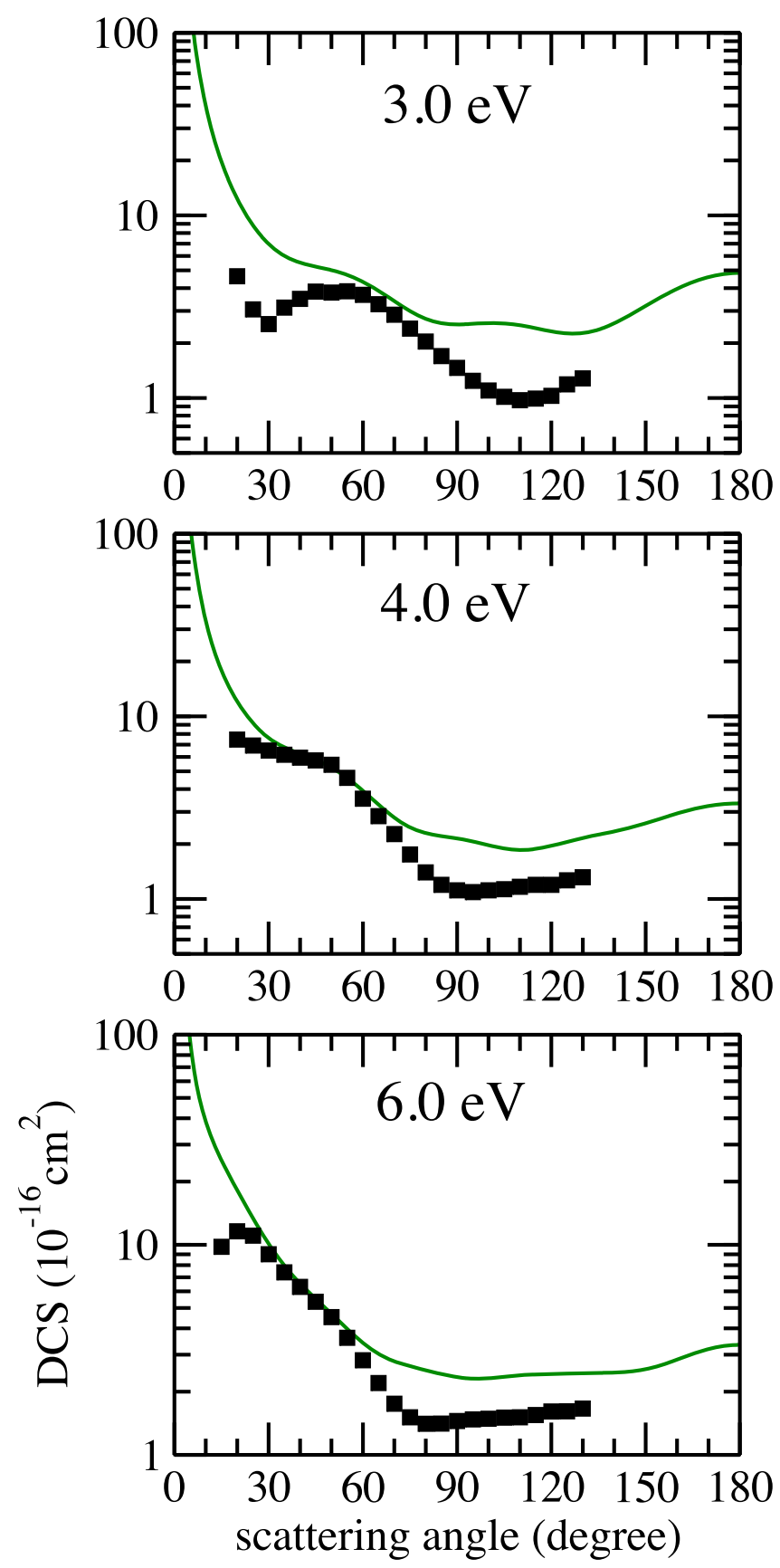

FIG. 4. Calculated differential cross sections for elastic scattering of electrons by chlorobenzene at $3.0 \mathrm{eV}, 4.0 \mathrm{eV}$, and $6.0 \mathrm{eV}$, obtained by the SMCPP method in the SEP+Born approximation (green line), compared with the experimental data available for benzene ${ }^{24}$ molecule (black squares).

essentially, by the shape resonances present at this energy range. Especially, we highlight the presence of a $\sigma^{*}$ resonance in $\mathrm{ClB}$, at around $3.0 \mathrm{eV}$, absent in benzene molecule.

Figures 5 and 6 present the theoretical and experimental DCSs for elastic scattering of electrons by $\mathrm{ClB}$ at $8 \mathrm{eV}$ and $10 \mathrm{eV}$, and at $20 \mathrm{eV}, 30 \mathrm{eV}$, and $50 \mathrm{eV}$ impact energies, respectively. The experimental scattering angles range from $7.0^{\circ}$ to $110^{\circ}$ and absolute values of the experimental cross sections are determined from the theoretical methods, from which the experimental data have been re-scaled at $30^{\circ}$ scattering angle. This normalization was done employing 

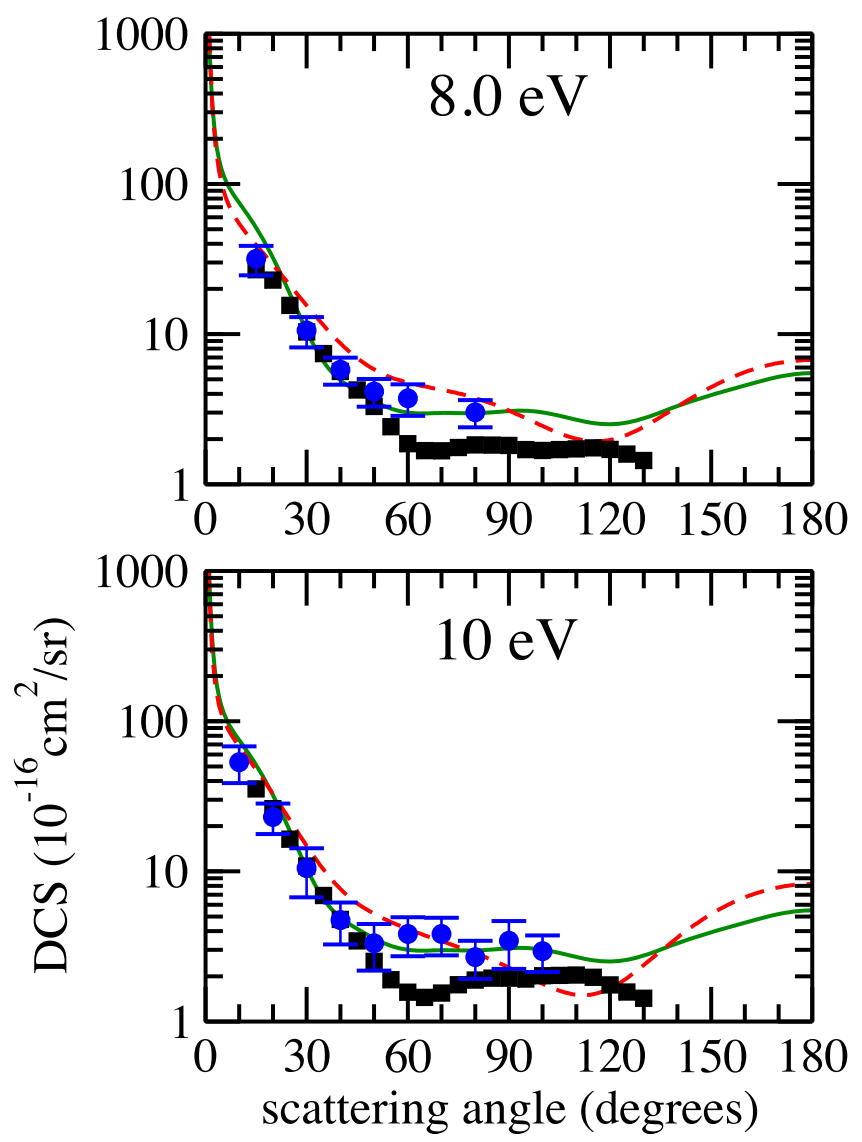

FIG. 5. Differential cross sections for elastic scattering of electrons by chlorobenzene at $8.0 \mathrm{eV}$ and $10 \mathrm{eV}$. Green solid line: present SMCPP results obtained in the SEP+Born approximation; dashed red line: present IAMSCAR results; blue circles: present experimental DCSs for chlorobenzene; black squares: experimental results for benzene. ${ }^{24}$

the SMCPP data, except at $50 \mathrm{eV}$, where the IAM-SCAR data were employed since this method is more suitable to represent scattering situation at higher energies. The experimental DCSs are tabulated in Table I. The theoretical SMCPP DCSs were obtained in the static-exchange plus polarization approximation at $8 \mathrm{eV}$ and $10 \mathrm{eV}$ and in the staticexchange approximation at $20 \mathrm{eV}, 30 \mathrm{eV}$, and $50 \mathrm{eV}$, where the polarization potential has little effect in the scattering process.

Here we note that $\mathrm{ClB}$ differential cross sections are dominated by strong increase in the forward scattering direction. This is essentially because the effects of the molecular dipole are most often seen at very small scattering angles. Note that the angular acceptance of the scattering apparatus $\left(<2^{\circ}\right)$ limits the ability of the experimental system to distinguish between unscattered and elastically or rotationally scattered electrons within the acceptance angle of the apparatus. We also compare the DCSs of $\mathrm{ClB}$ and benzene and found some similarities at $30 \mathrm{eV}$ and $50 \mathrm{eV}$, especially with the IAM-SCAR method.

An excellent agreement is observed between experimental and theoretical DCSs obtained with the SMCPP method, mainly at $8 \mathrm{eV}$ and $10 \mathrm{eV}$. There is also a good qualitative agreement at 20 and $30 \mathrm{eV}$ although at those energies the theoretical SMCPP DCSs are quantitatively higher than the
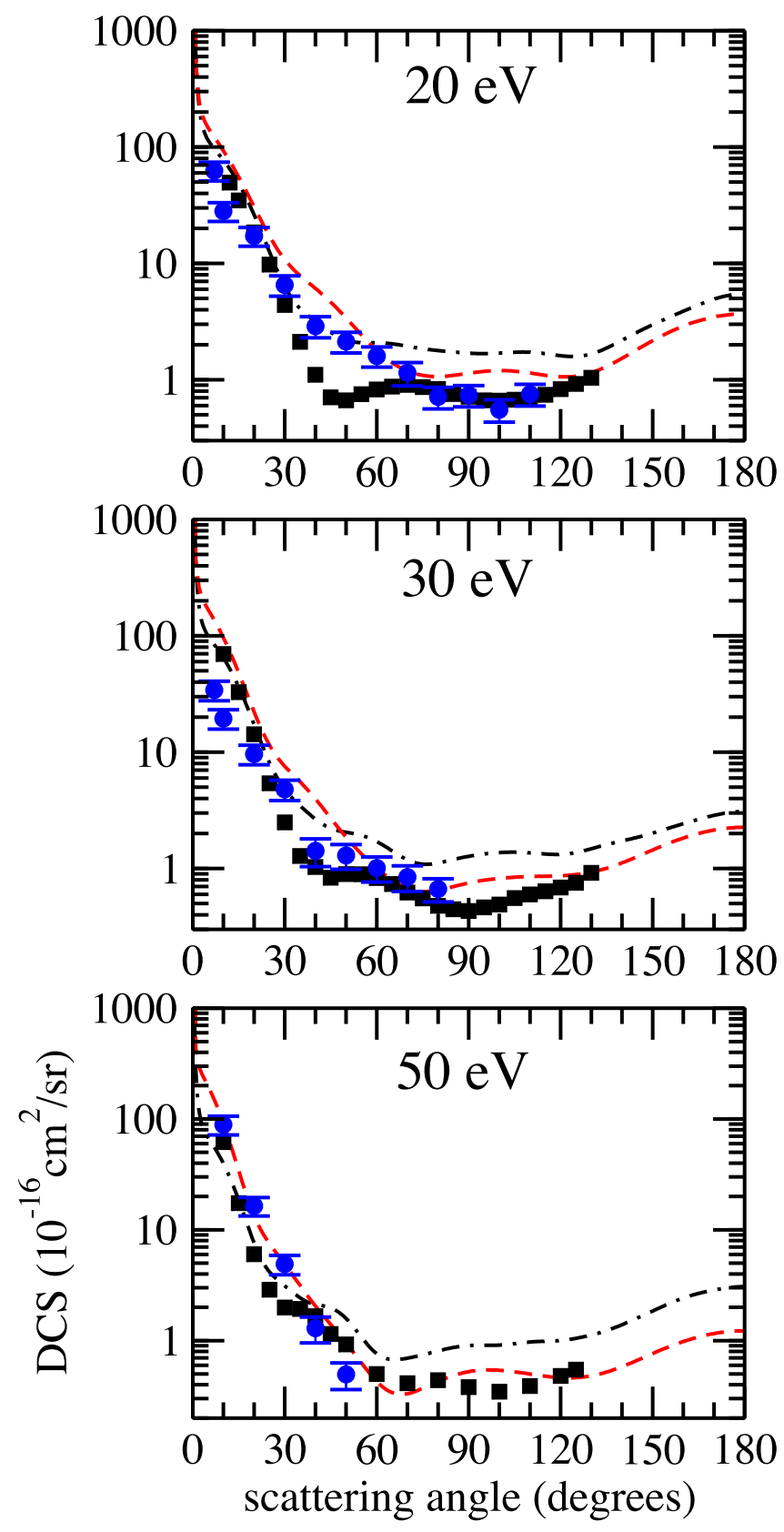

FIG. 6. Differential cross sections for elastic scattering of electrons by chlorobenzene at $20 \mathrm{eV}, 30 \mathrm{eV}$, and $50 \mathrm{eV}$. Black dotted-dashed line: present SMCPP results obtained in the SE+Born approximation; dashed red line: present IAM-SCAR results; blue circles: present experimental DCSs; black squares: experimental DCSs for benzene molecule at 20 and $30 \mathrm{eV}$ from Ref. 24, and at $50 \mathrm{eV}$ from Ref. 49.

experimental data. That is due to the purely elastic character of the present calculation. Opening inelastic channels, considered closed in our calculations, would allow flux loss from the elastic channel to the inelastic ones, lowering the elastic cross sections towards the experiment. ${ }^{48}$ Regarding the calculated IAM-SCAR DCSs, a better agreement with the present experimental DCSs in the electron energy range above $20 \mathrm{eV}$ is observed. At $50 \mathrm{eV}$, the experimental differential cross sections have been re-scaled at $30^{\circ}$ with the IAM-SCAR data, more suitable to represent the scattering situation at this energy. 
TABLE I. Tabulated experimental DCSs for elastic collisions of electrons with chlorobenzene molecule. The data are presented in $10^{-16} \mathrm{~cm}^{2} / \mathrm{sr}$ units.

\begin{tabular}{lrrrrr}
\hline \hline Angle $\left(^{\circ}\right)$ & $8.0 \mathrm{eV}$ & $10 \mathrm{eV}$ & $20 \mathrm{eV}$ & $30 \mathrm{eV}$ & $50 \mathrm{eV}$ \\
\hline 7 & & & 62.67 & 34.18 & 88.97 \\
10 & & 53.34 & 28.10 & 19.47 & 16.46 \\
15 & 31.69 & & & & \\
20 & & 23.02 & 17.22 & 9.63 & 4.90 \\
30 & 10.57 & 10.49 & 6.52 & 4.79 & 1.29 \\
40 & 5.79 & 4.73 & 2.89 & 1.42 & 0.50 \\
50 & 4.16 & 3.33 & 2.13 & 1.29 & \\
60 & 3.75 & 3.84 & 1.60 & 1.01 & \\
70 & & 3.84 & 1.15 & 0.84 & \\
80 & 3.02 & 2.69 & 0.71 & 0.66 & \\
90 & & 3.45 & 0.74 & & \\
100 & & 2.94 & 0.55 & & \\
110 & & & 0.75 & & \\
\hline \hline
\end{tabular}

\section{SUMMARY}

In this work, we reported a combined theoretical and experimental study on electron interactions with ClB. The theoretical ICS and DCSs were obtained with two different methods, SMCPP and IAM-SCAR, and the experimental DCSs were measured for impact energies from 8.0 up to $50 \mathrm{eV}$. From the low-energy SMCPP ICS, we reported shaperesonances characterized as $\pi^{*}$ at around $0.7 \mathrm{eV}, 0.76 \mathrm{eV}$, and $5.2 \mathrm{eV}$ belonging to the $B_{1}, A_{2}$, and $B_{1}$ symmetries, respectively, and a $\sigma^{*}$ resonance at $2.8 \mathrm{eV}$ in the $A_{1}$ symmetry. Our values agree well with ETS data and with the electron impact spectroscopy. A general good agreement was found between the calculated SMCPP and IAM-SCAR cross sections with experimental TCS available in the literature. Especially, we highlight the two resonant structures present in the experimental TCS at $0.75 \mathrm{eV}$ and $0.8 \mathrm{eV}$, from Refs. 22 and 23 , respectively, for the first structure, and at $2.5 \mathrm{eV}$ from Ref. 23 for the second structure, in very good agreement with the SMCPP ICS structures at 0.7 and $2.8 \mathrm{eV}$, and the excellent agreement of IAM-SCAR cross sections with experimental TCS for energies higher than $20 \mathrm{eV}$. The agreement between the two sets of calculated DCSs and the experimental data is in general very good. Especially, we highlight the excellent agreement between experimental and theoretical SMCPP DCSs at 8.0 and $10 \mathrm{eV}$ impact energy, and at higher energies between experimental and calculated IAM-SCAR data.

\section{ACKNOWLEDGMENTS}

A.S.B., M.T.N.V., S.d'A.S., and M.H.F.B. acknowledge the Brazilian Agency Coordenação de Aperfeiçoamento de Pessoal de Nível Superior (CAPES), under CAPES/FCT Programme (Process No. 23038.002465/2014-87). M.T.N.V., S.d'A.S., and M.H.F.B. acknowledge support from the Brazilian Agency Conselho Nacional de Desenvolvimento Científico e Tecnológico. M.H.F.B. acknowledges support from Finep (under project CT-Infra), and M.T.N.V. from São Paulo Research Foundation (FAPESP). A.S.B., S.d'A.S., and M.H.F.B. acknowledge computational support from Professor Carlos M. de Carvalho at LFTC-DFis-UFPR and at LCPAD-
UFPR and from CENAPAD-SP. F.F.S. acknowledges the Portuguese National Funding Agency FCT through researcher Contract No. IF-FCT IF/00380/2014 and together with P.LV. the research Grant No. UID/FIS/00068/2013. F.B. and G.G. acknowledge partial financial support from the Spanish Ministry MINECO (Project No. FIS2012-31230).

${ }^{1}$ B. Boudaïffa, P. Cloutier, D. Hunting, M. A. Huels, and L. Sanche, Science 287, 1658 (2000).

${ }^{2}$ X. Pan, P. Cloutier, D. Hunting, and L. Sanche, Phys. Rev. Lett. 90, 208102 (2003).

${ }^{3}$ C. Winstead and V. McKoy, Radiat. Phys. Chem. 77, 1258 (2008).

${ }^{4}$ I. Baccarelli, I. Bald, F. A. Gianturco, E. Illenberger, and J. Kopyra, Phys. Rep. 508, 1 (2011).

${ }^{5}$ E. Alizadeh, T. M. Orlando, and L. Sanche, Annu. Rev. Phys. Chem. 66, 379 (2015).

${ }^{6}$ J. Kopyra, A. Keller, and I. Bald, RSC Adv. 4, 6825 (2014).

${ }^{7}$ F. Kossoski, M. H. F. Bettega, and M. T. do N. Varella, J. Chem. Phys. 140, 024317 (2014).

${ }^{8}$ F. Kossoski and M. T. do N. Varella, Phys. Chem. Chem. Phys. 17, 17271 (2015).

${ }^{9}$ F. Kossoski, J. Kopyra, and M. T. do N. Varella, Phys. Chem. Chem. Phys. 17, 28958 (2015).

${ }^{10}$ A. S. Barbosa and M. H. F. Bettega, J. Chem. Phys. 139, 214301 (2013).

${ }^{11}$ P. Wardman, Clin. Oncol. 19, 397 (2007).

${ }^{12}$ A. Modelli, P. Bolognesi, and L. Avaldi, J. Phys. Chem. A 115, 10775 (2011).

${ }^{13}$ I. Nenner and G. J. Schulz, J. Chem. Phys. 62, 1747 (1975).

${ }^{14}$ J. K. Olthoff, J. A. Tossell, and J. H. Moore, J. Chem. Phys. 83, 5627 (1985).

${ }^{15}$ P. D. Burrow, A. Modelli, and K. D. Jordan, Chem. Phys. Lett. 132, 441 (1986).

${ }^{16}$ L. G. Christophorou, R. N. Compton, G. S. Hurst, and P. W. Reinhardt, J. Chem. Phys. 45, 536 (1966).

${ }^{17}$ J. Milhaud, Chem. Phys. Lett. 118, 167 (1985).

${ }^{18}$ K. L. Stricklett, S. C. Chu, and P. D. Burrow, Chem. Phys. Lett. 131, 279 (1986).

${ }^{19}$ R. V. Khatymov, M. V. Muftarkhov, and V. Mazunov, Rapid Commun. Mass Spectrom. 17, 2327 (2003).

${ }^{20}$ T. Skalický, C. Chollet, N. Pasquier, and M. Allan, Phys. Chem. Chem. Phys. 4, 3583 (2002).

${ }^{21}$ A. Modelli and M. Venuti, J. Phys. Chem. A 105, 5836 (2001).

${ }^{22}$ S. L. Lunt, D. Field, S. V. Hoffmann, R. J. Gulley, and J.-P. Ziesel, J. Phys. B 32, 2707 (1999).

${ }^{23}$ C. Makochekanwa, O. Sueoka, and M. Kimura, J. Chem. Phys 119, 12257 (2003).

${ }^{24}$ H. Cho, R. J. Gulley, K. Sunohara, M. Kitajima, L. J. Uhlmann, H. Tanaka, and S. J. Buckman, J. Phys. B 34, 1019 (2001).

${ }^{25}$ M. H. F. Bettega, A. P. P. Natalense, M. A. P. Lima, and L. G. Ferreira, J. Phys. B 36, 1263 (2003).

${ }^{26}$ K. Takatsuka and V. McKoy, Phys. Rev. A 24, 2473 (1981); 30, 1734 (1984).

${ }^{27}$ M. A. P. Lima, L. M. Brescansin, A. J. R. da Silva, C. Winstead, and V. McKoy, Phys. Rev. A 41, 327 (1990).

${ }^{28}$ M. H. F. Bettega, L. G. Ferreira, and M. A. P. Lima, Phys. Rev. A 47, 1111 (1993).

${ }^{29}$ R. F. da Costa, M. T. do N. Varella, M. H. F. Bettega, and M. A. P. Lima, Eur. Phys. J. D 69, 159 (2015).

${ }^{30}$ G. B. Bachelet, D. R. Hamann, and M. Schlüter, Phys. Rev. B 26, 4199 (1982)

${ }^{31}$ See http://cccbdb.nist.gov/ for the optimized geometries obtained at different levels of approximations and with different basis sets.

${ }^{32}$ M. W. Schmidt, K. K. Baldridge, J. A. Boatz, S. T. Elbert, M. S. Gordon, J. H. Jensen, S. Koseki, N. Matsunaga, K. A. Nguyen, S. J. Su, T. L. Windus, M. Dupuis, and J. A. Montgomery, J. Comput. Chem. 14, 1347 (1993).

${ }^{33}$ M. H. F. Bettega, A. P. P. Natalense, M. A. P. Lima, and L. G. Ferreira, Int. J. Quantum Chem. 60, 821 (1996).

${ }^{34}$ W. J. Hunt and W. A. Goddard III, Chem. Phys. Lett. 3, 414 (1969)

${ }^{35}$ F. Kossoski and M. H. F. Bettega, J. Chem. Phys. 138, 234311 (2013).

${ }^{36}$ F. Blanco and G. García, Phys. Lett. A 360, 707 (2007).

${ }^{37}$ F. Blanco, J. Rosado, A. Illana, and G. García, Phys. Lett. A 374, 4420 (2010)

${ }^{38}$ P. Limão-Vieira, M. Horie, H. Kato, M. Hoshino, F. Blanco, G. García, S. J. Buckman, and H. Tanaka, J. Chem. Phys. 135, 234309 (2011).

${ }^{39}$ H. Kato, A. Suga, M. Hoshino, F. Blanco, G. García, P. Limão-Vieira, M. J. Brunger, and H. Tanaka, J. Chem. Phys. 136, 134313 (2012). 
${ }^{40}$ H. Kato, K. Anzai, T. Ishihara, M. Hoshino, F. Blanco, G. García, P. LimãoVieira, M. J. Brunger, S. J. Buckman, and H. Tanaka, J. Phys. B: At., Mol. Opt. Phys. 45, 095204 (2012).

${ }^{41}$ H. Murai, Y. Ishijima, T. Mitsumura, Y. Sakamoto, H. Kato, M. Hoshino, F. Blanco, G. García, P. Limão-Vieira, M. J. Brunger, S. J. Buckman, and H. Tanaka, J. Chem. Phys. 138, 054302 (2013).

${ }^{42}$ M. Hoshino, P. Limão-Vieira, K. Anzai, H. Kato, H. Cho, D. Mogi, T. Tanioka, F. Ferreira da Silva, D. Almeida, F. Blanco, G. García, O. Ingolfsson, and H. Tanaka, J. Chem. Phys. 141, 124302 (2014).

${ }^{43}$ F. Motte-Tollet, M.-J. Hubin-Franskin, and J. E. Collin, J. Chem. Phys. 97, 7314 (1992).

${ }^{44}$ M. A. Khakoo, P. V. Johnson, I. Ozkay, P. Yan, S. Trajmar, and I. Kanik, Phys. Rev. A 71, 062703 (2005).
${ }^{45}$ C. Winstead and V. McKoy, Phys. Rev. Lett. 98, 113201 (2007)

${ }^{46}$ CRC Handbook of Chemistry and Physics, 85th ed., edited by D. R. Lide (CRC, Boca Raton, 2005).

${ }^{47}$ E. M. de Oliveira, R. F. da Costa, S. d'A. Sanchez, A. P. P. Natalense, M. H. F. Bettega, M. A. P. Lima, and M. T. do N. Varella, Phys. Chem. Chem. Phys. 15, 1682 (2013).

${ }^{48}$ R. F. da Costa, M. H. F. Bettega, M. A. P. Lima, M. C. A. Lopes, L. R. Hargreaves, G. Serna, and M. A. Khakoo, Phys. Rev. A 85, 062706 (2012).

${ }^{49}$ I. P. Sanches, R. T. Sugohara, L. Rosani, M.-T. Lee, and I. Iga, J. Phys. B 41, 185202 (2008).

${ }^{50}$ M. H. F. Bettega, C. Winstead, and V. McKoy, J. Chem. Phys. 112, 8806 (2000).

${ }^{51}$ F. A. Gianturco and R. R. Lucchese, J. Chem. Phys. 108, 6144 (1998). 